Helle Borgstrøm Hager*, Nils Bolstad, David J. Warren, Marianne Vindal Ness, Birgitte Seierstad and Morten Lindberg

\title{
Falsely markedly elevated 25-hydroxyvitamin D in patients with monoclonal gammopathies
}

https://doi.org/10.1515/cclm-2020-1411

Received September 18, 2020; accepted October 11, 2020; published online October 30, 2020

\section{Abstract}

Objectives: Monoclonal immunoglobulins can cause interference in many laboratory analyses. During a 4 month period we observed seven patients with monoclonal disease and falsely extremely elevated 25-hydroxyvitamin D (25(OH)D) results above $160 \mathrm{ng} / \mathrm{mL}$ (>400 nmol/L) measured using an immunoassay from Abbott Diagnostics. Based on these findings, we studied the occurrence of falsely elevated 25(OH)D in samples with paraproteins and investigated possible mechanisms of the observed interference.

Methods: $25(\mathrm{OH}) \mathrm{D}$ was analyzed using the Architect $\mathrm{i} 2000$ platform from Abbott Diagnostics and a higher order method, liquid chromatography-mass spectrometry (LC-MS/MS), in serum samples from 50 patients with known monoclonal disease. Patients with falsely elevated 25(OH)D were included in further studies to elucidate the cause of interference. Spuriously elevated results were in addition analyzed on two alternative platforms (Siemens and Roche).

Results: Falsely elevated 25(OH)D levels were present in eight patients on the Abbott analyzer and one on the Siemens platform. Results from Roche were comparable with LC-MS/MS. Additional investigations excluded elevated concentrations of rheumatoid factor and heterophilic antibodies as the cause of interference in the Abbott assay.

*Corresponding author: Helle Borgstrøm Hager, M.D., Department of Medical Biochemistry, Vestfold Hospital Trust, PO Box 2168, 3103 Tønsberg, Norway, Tel. (office): +4733343053, Fax: +4733343942, E-mail: helle.hager@siv.no

Nils Bolstad and David J. Warren, Department of Medical Biochemistry, Oslo University Hospital - Radiumhospitalet, Oslo, Norway

Marianne Vindal Ness and Morten Lindberg, Department of Medical Biochemistry, Vestfold Hospital Trust, Tønsberg, Norway Birgitte Seierstad, Department of Laboratory Diagnostics, Vestre Viken Hospital Trust, Drammen, Norway
Conclusions: Laboratories should be aware of the risk of falsely elevated 25(OH)D in samples run on the Architect analyzer from patients with monoclonal disease. Highly elevated vitamin D results should be diluted and if the dilution is non-linear, rerun by a different method, preferably LC-MS/MS. In patients with spuriously elevated 25(OH)D without known monoclonal disease, the laboratory should consider requesting protein electrophoresis to exclude paraprotein interference.

Keywords: analytical interference; artefact; humans; immunoassay; monoclonal protein; paraproteins; 25-hydroxyvitamin D2.

\section{Introduction}

Paraproteins occur in urine or plasma in multiple myeloma, Waldenstrom's macroglobulinaemia, plasmacytoma, amyloidosis, and monoclonal gammopathy of undetermined significance (MGUS). It is known that paraproteins may affect laboratory results due to preanalytical or analytical interference [1]. Different mechanisms may be responsible for the interference, e.g., increased turbidity [2], increased viscosity [3], chemical interference [4] or binding to proteins such as antibodies or analytes in immunoassays [5].

Serum 25-hydroxyvitamin D $(25(\mathrm{OH}) \mathrm{D})$ is routinely analyzed using competitive immunoassays or liquid chromatography coupled with tandem mass spectrometry (LC-MS/MS)). LC-MS/MS is more accurate and specific and is recognized as being a higher order method [6], but it is more labor intensive than the immunoassays. Automated immunoassays are therefore more commonly in use. Unfortunately, they are also more prone to interference.

During a period of 4 months in summer 2016, we discovered seven patients with falsely markedly elevated 25(OH)D results from our immunoassay performed on the Abbott Architect i2000sr (Abbott Laboratories, Abbott Park, Illinois, USA). Results were above the upper assay range of $160 \mathrm{ng} / \mathrm{mL}$ (400 nmol/L) and the samples did not dilute linearly, suggesting interference. Analysis was repeated by LC-MS/MS, showing lower values in the range 
of $<4-30 \mathrm{ng} / \mathrm{mL}$. All seven patients had monoclonal gammopathies, either multiple myeloma or Walderstrom's macroglobulinemia. Two of them had no known monoclonal disease, and investigations that revealed a paraprotein were ordered by the laboratory doctor due to suspicion raised by the spuriously elevated 25(OH)D. We informed Abbott Diagnostics in October 2016 about the observed discrepant vitamin D results, affecting 3 different reagents lots in the period.

We decided to investigate whether falsely elevated vitamin D measured on the Abbott Architect was common in samples from patients with monoclonal gammopathies. We also wanted to study whether immunoassays from other companies were susceptible to this interference, and attempt to identify the cause of the interference.

\section{Materials and methods}

\section{Study population}

Serum samples from 50 patients with monoclonal immunoglobulin concentration of $5 \mathrm{~g} / \mathrm{L}$ or above were consecutively frozen at $-20^{\circ} \mathrm{C}$ after performance of capillary electrophoresis. When collection was completed, all samples were thawed and $25-\mathrm{OH}$ vitamin D was analyzed using two different methods, competitive immunoassay from Abbott Architect and LC/MS-MS from Agilent.

The samples were then mixed with polyethylene glycol 6000 (PEG) solution 25\% from Merck (dilution 1:1) and incubated at room temperature for 10 min using a gentle shaker. PEG precipitation test has the ability to remove immunoglobulins and is widely used to detect pseudohyperprolactinemia caused by macroprolactin, but can also be used to remove immunoglobulins in general [7]. The suspension was centrifuged at $4000 \mathrm{~g}$ for $10 \mathrm{~min}$ and the supernatant was rerun on both methods.

The patients who had falsely elevated vitamin D on the Abbott Architect constituted our study population and were asked to donate two extra vacutainers at venous blood sampling in connection with their next doctor's appointment. Serum was centrifuged within an hour and frozen in aliquots at $-70^{\circ} \mathrm{C}$. The study was approved by the Data Protection Official for Research for all the Norwegian universities. Written informed consent was obtained from all participants.

Eight serum samples from patients with monoclonal disease, but no aberrant vitamin $D$ results, were randomly selected as controls from the 50 samples above. Leftover serum after performance of capillary electrophoresis was frozen at $-70^{\circ} \mathrm{C}$.

\section{Laboratory measurement procedures}

25(OH)D was analyzed on Architect i2000SR from Abbott Diagnostics, USA with the latest generation of the Architect 25(OH)D assay (product 5P02), launched in 2015 and standardized to NIST SRM 2972 [8]. Abbott's vitamin D analysis is a competitive chemiluminiscent microparticle immunoassay that uses rabbit monoclonal antibodies coated onto paramagnetic particles and an acridinium labeled conjugate. Pretreatment dissociates 25(OH)D from vitamin $\mathrm{D}$ binding protein, and 25(OH)D competes with exogenous labeled 25(OH)D for binding to assay specific antibodies. The measuring range is $3.4-160 \mathrm{ng} / \mathrm{mL}(8.5-400 \mathrm{nmol} / \mathrm{L})$ and the experienced analytical coefficient of variation (CV) is $7 \%$. According to the package insert, possible interferences in the assay are high concentrations of rheumatoid factor, human heterophilic antibodies, antirabbit antibodies (HARAs), and triglyceridemia.

25(OH)D was also analyzed on a liquid chromatography tandem mass spectrometer (LC-MS/MS) with calibrators traceable to NIST SRM 2972a. The measuring range is $3.4-240 \mathrm{ng} / \mathrm{mL}(8.5-600 \mathrm{nmol} / \mathrm{L}), \mathrm{CV}$ $7 \%$. The Laboratory is accredited according to ISO 15189 and participates in the vitamin D external quality assessment scheme from DEQAS.

To assess the possible interference of HARAs or heterophilic antibodies, we performed interference assays [9] on all eight samples from patients having falsely elevated 25(OH)D and the eight controls, using monoclonal murine IgG1, polyclonal bovine IgG and polyclonal rabbit IgG both as solid phase and tracer proteins.

To investigate if other immunoassays also were vulnerable to interference, we performed analysis of $25(\mathrm{OH}) \mathrm{D}$ in sera from the patients with spuriously elevated 25(OH)D using two different automated immunoassay methods: Elecsys vitamin D on Cobas e801 from Roche Diagnostics GmbH, Mannheim, Germany and Advia Centaur from Siemens Healthcare Diagnostics, Tarrytown, USA.

Analysis of Rheumatoid factor was performed in samples from the same patients to exclude interference due to high concentration of rheumatoid factor, using Quanta Lite RF IgM ELISA from Inova Diagnostics.

Because 25(OH)D is dissociated from vitamin D-binding protein in a pretreatment procedure on the Abbott method, we also wanted to exclude the presence of deviant concentrations of vitamin D binding protein in our study patients. Analysis of vitamin D binding protein in the patients and the eight controls without aberrant vitamin $D$ results was performed using an in-house competitive radioimmunoassay at The Hormone Laboratory, Oslo University Hospital.

Finally, we attempted to elucidate the cause of the interference. Iodine-125-labeled human IgA was added to one of the patient samples in order to control which fraction contained the monoclonal component (in this case IgA kappa). The sample was centrifuged in a Vivaspin 20 centrifugal concentrator (Sartorius, Goettingen, Germany) with a $50 \mathrm{kDa}$ molecular weight cut-off until half the volume had passed through the membrane. The filtrate and the concentrate were then reanalyzed on the Architect assay. The concentrate was subsequently applied to a $1 \mathrm{~mL}$ HiTrap Protein G HP column (GE Healthcare Life Sciences, Chicago, IL). The flowthrough, containing vitamin D binding globulin and IgA, and the eluate, containing IgG, were reanalyzed in the Architect assay.

\section{Results}

Of the 50 serum samples from patients with monoclonal disease, 29 samples contained IgG M-components (10 lambda and 19 kappa), concentration range from 5.9 to $51.0 \mathrm{~g} / \mathrm{L}$. Thirteen were IgM M-components (2 lambda and 11 kappa), concentration range from 6.4 to $42.3 \mathrm{~g} / \mathrm{L}$ and eight were IgA M-components (6 lambda and 2 kappa), concentration range from 7.8 to $28.5 \mathrm{~g} / \mathrm{L}$. Among the 50 
samples, four had falsely elevated 25(OH)D levels, three of these from patients already identified. One sample was excluded due to a missing 25(OH)D value. The remaining 45 samples showed good correlation between the Abbott and the LC-MS/MS-method (results not shown). From these 45 samples, we randomly selected eight as controls. Their median concentration of the M-component was $14 \mathrm{~g} / \mathrm{L}$ (range from $7.8 \mathrm{~g} / \mathrm{L}$ to $45.1 \mathrm{~g} / \mathrm{L}$ ).

\section{Characteristics of patients with falsely elevated 25(OH)D}

In total, we found eight patients with paraproteins having falsely markedly elevated 25(OH)D results (Table 1). Monoclonal IgM was the most prevalent immunoglobulin. The patients had either multiple myeloma (three women and one man) or Waldenstrom's macroglobulinaemia (four men). Their median concentration of the M-component was $29.9 \mathrm{~g} / \mathrm{L}$ (range 14.5-51.0). The median age was 77 (range 65-95 years). The extent of their disease varied from smoldering myeloma to refractory multiple myeloma with short life expectancy. Investigation of their medical records did not show any similarities regarding comorbidities, present or past medication.

None of the serum samples from the patients with spuriously elevated vitamin D were lipemic and their median vitamin $\mathrm{D}$ binding protein concentration did not differ from the controls. The median vitamin $\mathrm{D}$ binding protein concentration in the patients was 3.9 $\mu \mathrm{mol} / \mathrm{L}$ (range 2.9-5.7), whereas the controls' median concentration was $3.7 \mu \mathrm{mol} / \mathrm{L}$ (range 2.9-4.5). Analyses of rheumatoid factor in sera from our study patients revealed one weak positive (11 U/L, cutoff $10 \mathrm{U} / \mathrm{L}$ ), the remaining seven were negative.
Results from analysis of 25(OH)D on different platforms for the patients are shown in Figure 1. Only one of the eight patients with spuriously elevated vitamin D using the immunoassay from Abbott, had falsely elevated results on Siemens, whereas none of them displayed spuriously elevated result on Roche.

\section{Results after treatment with PEG}

After PEG precipitation the samples from the patients and the 44 controls were rerun on the Architect and the LC-MS/MS. The values obtained on both methods after PEG precipitation were not statistically different. The median recovery on LC-MS/MS was $81 \%$ in the patients and $92 \%$ in the controls. Concentrations of $25(\mathrm{OH}) \mathrm{D}$ using immunoassay from Abbott and LC-MS/MS before and after PEG precipitation is shown for the eight study patients in Figure 2 and the eight controls in Figure 3.

\section{Heterophilic and anti-animal antibodies}

Samples from the study patients and the controls were assayed for heterophilic and anti-animal antibodies by immunometric assay. Monoclonal murine IgG1, polyclonal bovine IgG and polyclonal rabbit IgG were used as both solid phase and tracer proteins. Few samples in either group gave convincing signals in any assays, and no systematic differences between cases and controls were observed. In the assay using rabbit IgG, one case patient and two control patients gave weak positive signals, but pre-blocking these samples with aggregated rabbit IgG gave no effect on the results for vitamin D. In conclusion, our investigations gave no convincing evidence for

Table 1: Serum 25-hydroxyvitamin D (25(OH)D) concentrations measured by two different methods, type and concentration of monoclonal immunoglobulin and diagnosis in the eight study patients.

\begin{tabular}{|c|c|c|c|c|c|}
\hline $\begin{array}{l}\text { Patient } \\
\text { number }\end{array}$ & $\begin{array}{r}25(\mathrm{OH}) \mathrm{D} \\
\text { Abbott architect, } \\
\mathrm{ng} / \mathrm{mL}\end{array}$ & $\begin{array}{r}25(\mathrm{OH}) \mathrm{D} \\
\mathrm{LC}-\mathrm{MS} / \mathrm{MS}, \\
\mathrm{ng} / \mathrm{mL}\end{array}$ & $\begin{array}{l}\text { Type of monoclonal } \\
\text { immunoglobulin }\end{array}$ & $\begin{array}{r}\begin{array}{r}\text { Concentration } \\
\text { of monoclonal }\end{array} \\
\text { immunoglobulin, } \mathrm{g} / \mathrm{L}\end{array}$ & Diagnosis \\
\hline 1 & $>160$ & 34 & IgM Lambda & 14.5 & $\begin{array}{l}\text { Waldenstrom's } \\
\text { macroglobulinaemia }\end{array}$ \\
\hline 2 & 138 & 38 & IgM Карра & 16.2 & $\begin{array}{l}\text { Waldenstrom's } \\
\text { macroglobulinaemia }\end{array}$ \\
\hline 3 & 150 & 40 & IgG Kappa & 22.0 & Smoldering myeloma* \\
\hline 4 & 160 & $<4.0$ & IgG Kappa & 26.4 & Multiple myeloma* \\
\hline 5 & $>160$ & 25 & IgM Карра & 33.6 & $\begin{array}{l}\text { Waldenstrom's } \\
\text { macroglobulinaemia }\end{array}$ \\
\hline 6 & $>160$ & 37 & IgG Lambda & 34.2 & Multiple myeloma \\
\hline 7 & $>160$ & 6.4 & IgA Карра & 41.0 & Multiple myeloma \\
\hline 8 & 99 & 27 & IgG Lambda & 51.0 & Multiple myeloma \\
\hline
\end{tabular}

The diagnoses marked by an asterix were detected due to discrepant vitamin $\mathrm{D}$ results. 


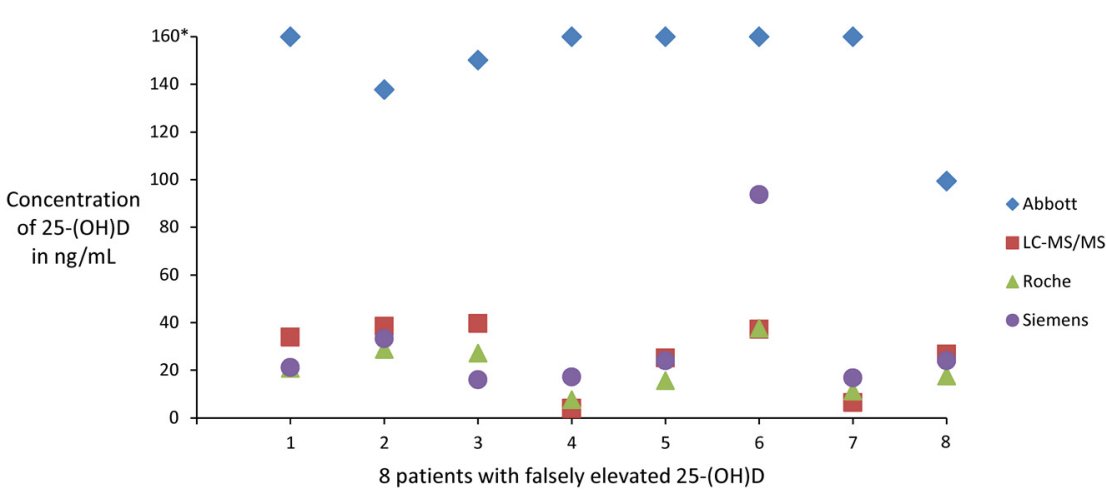

Figure 1: Concentrations of 25-hydroxyvitamin $\mathrm{D}(25(\mathrm{OH}) \mathrm{D})$ from the eight study patients measured by four different methods, using immunoassays from Abbott, Roche and Siemens and LC-MS/MS.

${ }^{*}$ Concentrations above $160 \mathrm{ng} / \mathrm{mL}$ are expressed as $160 \mathrm{ng} / \mathrm{mL}$ on the $\mathrm{Y}$-axis.

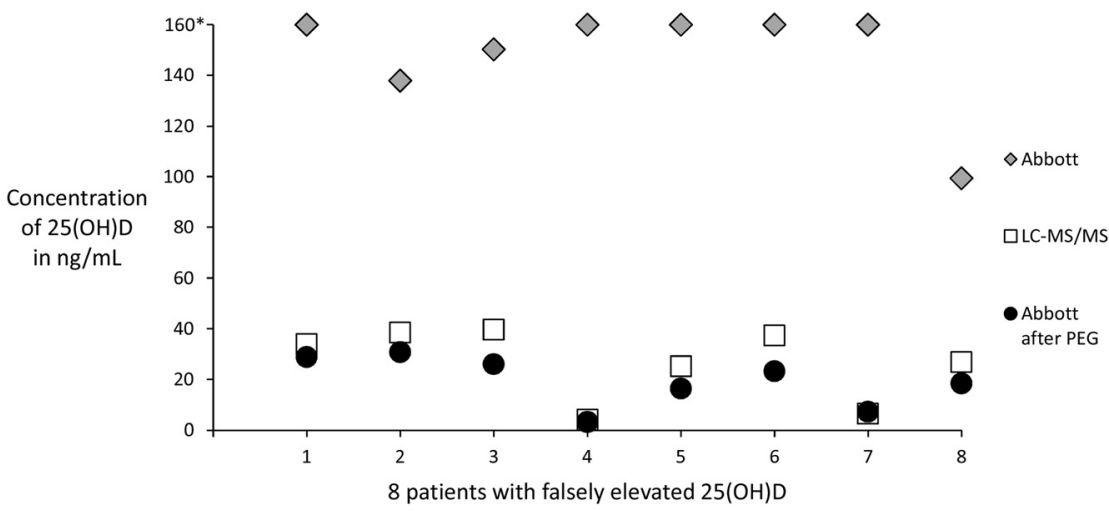

Figure 2: Concentrations of 25-hydroxyvitamin $\mathrm{D}(25(\mathrm{OH}) \mathrm{D})$ from the eight study patients measured by two different methods, using immunoassay from Abbott Diagnostics and LC-MS/MS. In addition, concentrations of 25(OH)D on the Abbott platform after polyethylene (PEG) precipitation is shown.

*Concentrations above $160 \mathrm{ng} / \mathrm{mL}$ are expressed as $160 \mathrm{ng} / \mathrm{mL}$ on the $\mathrm{Y}$-axis.

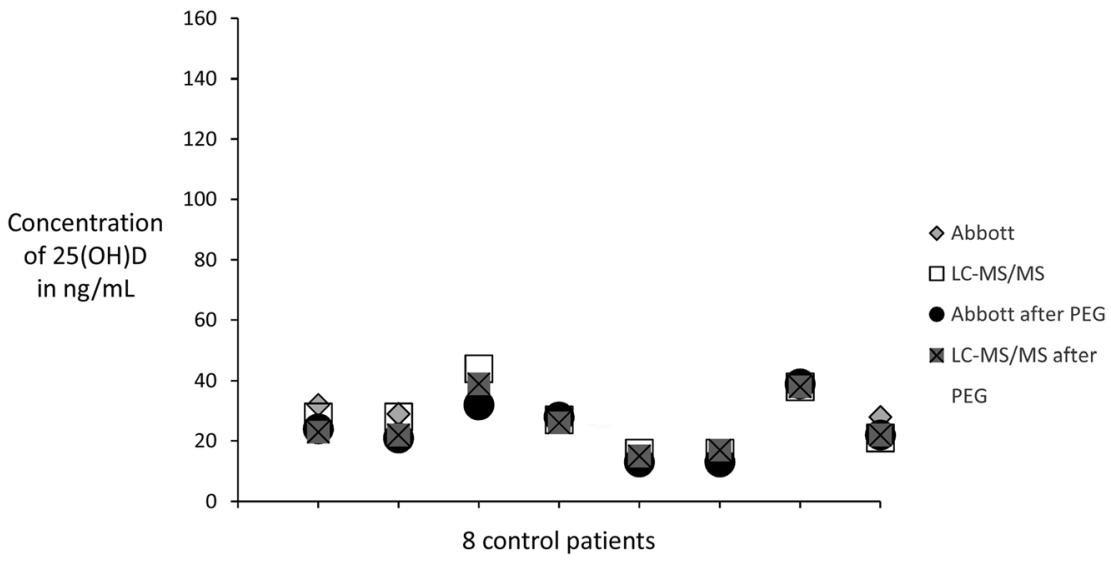

Figure 3: Concentrations of 25-hydroxyvitamin D (25(OH)D) from eight control patients having monoclonal disease measured by two different methods, using immunoassay from Abbott Diagnostics and LC-MS/MS. In addition, concentrations of $25(\mathrm{OH}) \mathrm{D}$ on the Abbott platform and on LC-MS/MS after polyethylene (PEG) precipitation are shown.

interference due to heterophilic antibodies or anti-rabbit antibodies.

\section{Results from attempts to determine the cause of interference}

Iodine-125-labeled human IgA was added to one of the patients' samples (patient number 7 in Table 1) in order to control which fraction contained the monoclonal component. After centrifugation in a Vivaspin 20 centrifugal ultrafilter, the flow-through, containing smaller molecules including vitamin $\mathrm{D}$ (385 Da), did not contain detectable free vitamin $\mathrm{D}$ when measured in the Architect assay. The retentate, containing larger molecules including immunoglobulins $(>150 \mathrm{kDa})$ and vitamin D-binding protein (58 kDa), gave an elevated result $(87 \mathrm{ng} / \mathrm{mL}$ ) in the Architect assay. After the retentate was applied on a HiTrap Protein G HP column, the flow-through (IgA and vitamin D-binding protein) gave a result of 30.6 in the Architect assay when corrected for dilution. The eluate, containing IgG, did not give any signal in the Architect assay. 


\section{Discussion}

We identified eight patients with falsely markedly elevated 25(OH)D measured on the Abbott Architect. All of these individuals had monoclonal gammopathies, implying that paraproteins were most likely involved in the interference. Herein we describe our attempts to identify the cause of spuriously elevated vitamin D.

Heterophilic antibodies, HARAs and lipemia are, in addition to rheumatoid factor, possible causes of interference in the $25(\mathrm{OH}) \mathrm{D}$ analysis, according to the package insert from Abbott. None of our samples from the study patients were lipemic and we found no evidence of rheumatoid factor, heterophilic antibodies or HARA interference.

The paraproteins in the eight patients might have caused macrocomplexes or macro-vitamin D. Macrocomplexes are well-documented as cause of falsely elevated results in assays for hormones [10], enzymes [11, 12] and vitamin $B_{12}$ [13]. However, macro-vitamin $D$ has to our knowledge not been described. Furthermore, the eight samples that showed falsely elevated results on the Abbott analyzer, did not display interference on the Roche platform, and only one interfered on the Siemens platform.

The incidence of paraprotein interference is unknown as there are few publications on monoclonal immunoglobulin interference in automated vitamin D-assays. One case report describes a falsely elevated concentration of vitamin D in a patient with a monoclonal peak of immunoglobulin $\mathrm{G}$ using Liaison XL from Diasorin [14]. Another case report concerns possible interference by an IgG paraprotein in the vitamin D assay from Abbott Architect [15]. Since this patient also suffered from rheumatoid arthritis, the authors argued that interference from rheumatoid factor might be the cause of the discordant result.

In our efforts to characterize the interference in this study, a major challenge was that even modest dilutions of the samples reduced or removed the falsely elevated signals in the Abbott 25(OH)D-assay. Dilutions are very difficult to avoid in the methods that could have helped us identify the source of interference. Although we were unable to prove it, we believe the most likely cause of interference in these samples is the precipitation of the monoclonal component when subjected to the dissociation agent added to release $25(\mathrm{OH}) \mathrm{D}$ from its binding protein in the assay. In this regard it is noteworthy that the assays that showed some interference both use 8-anilino1-naphthalenesulfonic acid (ANSA) as releasing agent albeit with differing buffer components. In contrast, the Roche assay, which does not display paraprotein interference, uses a unique system based on a strong reducing agent (DTT) and high pH. Antibody aggregation is influenced by both extrinsic (environment, protein concentration etc.,) and intrinsic (protein sequence and structure) factors. Thus, we hypothesize that the ability of ANSA to stabilize partially unfolded proteins may facilitate aggregation of a subset of paraproteins especially under the buffer conditions used in the Abbott 5P02 assay.

We performed 25(OH)D on the Abbott Architect i2000sr using the 3L52 assay kit from 2011 until spring 2016 without experiencing any erroneous results due to interferences. Up to 2016, only 21 samples out of approximately 100,000 analyses had elevated values above the potentially toxic threshold of $140 \mathrm{ng} / \mathrm{mL}$ (350 nmol/L) [16]. Twenty of these could be explained by overdosing of supplements. In 2016 we implemented a new and improved generation of the 25(OH)D Abbott Architect reagents, product 5P02, standardized to a new traceable reference standard, which replaced the 3L52 assay [8]. This method was thoroughly validated recently [17]. Our results suggest that samples containing monoclonal immunoglobulins should be included in future validation of 25(OH)D assays. Of notice, the selection of study samples from falsely elevated results run on the Abbott method, might have introduced a bias. We have not investigated the occurrence of falsely elevated 25(OH)D using other immunoassays. Seven of the eight patients with falsely markedly elevated 25(OH)D were identified during a period of 4 months in summer 2016. Whether the observed problem of interference is related to the new generation of reagents, is at present unclear. Indeed, the cluster of falsely elevated $25(\mathrm{OH}) \mathrm{D}$ results following the introduction of the 5P02 assay might have been purely coincidental.

During 2016 we undertook capillary electrophoresis on 252 different patients having monoclonal immunoglobulins in serum, but only 67 of them (27\%) had their vitamin D concentration checked. Thus, we cannot say how prevalent falsely elevated vitamin D results are among patients with monoclonal disease. Among our patients with known paraproteins, at least eight, equivalent to $3.2 \%$, had falsely elevated 25(OH)D. Multiple myeloma has been estimated to account for $1.7 \%$ of all malignancies and $10 \%$ of all hematological malignancies in the United States in 2017 [18]. Monoclonal gammopathy of undetermined significance (MGUS) was found in 3.2\% of persons 50 years of age or older, $5.3 \%$ of persons 70 years of age or older and $7.5 \%$ among those 85 years of age or older in a large population 
study among residents of Olmsted County, Minnesota [19]. Although all the patients we found who had falsely elevated 25(OH)D had myeloma or Waldenstrom's macroglobulinaemia, this does not exclude that patients having MGUS might also be affected by interference. If so, falsely elevated 25(OH)D can potentially affect a substantial number of patients.

\section{Conclusions}

To our knowledge, we are the first to describe falsely elevated 25-hydroxyvitamin $D$ results in several patients due to interference of monoclonal immunoglobulins. Falsely highly elevated 25-OH vitamin D using Abbott's immunoassay was discovered in eight patients during 2016 having multiple myeloma or Morbus Walderstrom. Our investigations did not reveal the precise cause of the interference. However, PEG precipitation removed the problem, and we suspect the monoclonal immunoglobulins to have caused the interference. Why these eight, and not all paraproteins, gave falsely elevated $25(\mathrm{OH})$ D-values, remains to be explained. Abbott's vitamin D assay on Architect has not been changed since 2016, and laboratories using the assay should be aware of this possible interference with paraproteins. Two of the eight patients in our study (patient number 4 and 7 in Table 1) would not have been diagnosed as vitamin $\mathrm{D}$ deficient if the falsely elevated results had not been disclosed. Highly elevated vitamin D results should be diluted and if the dilution is non-linear, rerun by a different method, preferably LC-MS/MS. PEG precipitation might be an alternative if chromatography is not available. In patients with markedly elevated 25(OH)D and no known monoclonal gammopathy, the laboratory should consider adding protein electrophoresis to exclude paraprotein interference. If falsely elevated $25-\mathrm{OH}$ vitamin D results are reported, patients risk going through unnecessary investigations and possibly to have an incorrect diagnosis and erroneous treatment.

Acknowledgments: The skilled technical assistance of Janne Myrsve Solheim at Department of Medical Biochemistry at Vestfold Hospital Trust is gratefully acknowledged.

Research funding: None declared.

Author contributions: All authors have accepted responsibility for the entire content of this manuscript and approved its submission.

Competing interests: Authors state no conflict of interest.
Informed consent: Informed consent was obtained from all individuals included in this study.

Ethical approval: The study was approved by the Data Protection Official for Research for all the Norwegian universities.

\section{References}

1. King RI, Florkowski CM. How paraproteins can affect laboratory assays: spurious results and biological effects. Pathology 2010; 42:397-401

2. Smogorzewska A, Flood JG, Long WH, Dighe AS. Paraprotein interference in automated chemistry analyzers. Clin Chem 2004; 50:1691-3.

3. Chan KM, Ladenson JH. Sample viscosity can be a source of analytical error when discrete sampler-dilutors are used. Clin Chem 1981;27:1896-8.

4. Datta $P$, Graham GA, Schoen I. Interference by IgG paraproteins in the Jaffe method for creatinine determination. Am J Pathol 1986; 85:463-8.

5. Luzzi VI, Scott MG, Gronowski AM. Negative thyrotropin assay interference associated with an IgGkappa paraprotein. Clin Chem 2003;49:709-10.

6. Tai SS, Bedner M, Phinney KW. Development of a candidate reference measurement procedure for the determination of 25hydroxyvitamin D3 and 25-hydroxyvitamin D2 in human serum using isotope-dilution liquid chromatography-tandem mass spectrometry. Anal Chem 2010;82:1942-8.

7. Ismail AA. A radical approach is needed to eliminate interference from endogenous antibodies in immunoassays. Clin Chem 2005; 51:25-6.

8. Hutchinson K, Healy M, Crowley V, Louw M, Rochev Y. Verification of Abbott 25-OH-vitamin D assay on the architect system. Pract Lab Med 2017;7:27-35.

9. Boscato LM, Stuart MC. Incidence and specificity of interference in two-site immunoassays. Clin Chem 1986;32:1491-5.

10. Ismail AA, Walker PL, Barth JH, Lewandowski KC, Jones R, Burr WA. Wrong biochemistry results: two case reports and observational study in 5310 patients on potentially misleading thyroidstimulating hormone and gonadotropin immunoassay results. Clin Chem 2002;48:2023-9.

11. Moriyama T, Tamura S, Nakano K, Otsuka K, Shigemura M, Honma N. Laboratory and clinical features of abnormal macroenzymes found in human sera. Biochim Biophys Acta 2015;1854:658-67.

12. Moriyama T, Nobuoka M, Makino M. Incidence and properties of aspartate aminotransferase-immunoglobulin complexes in patients with a high serum aspartate to alanine aminotransferase ratio. Clin Chim Acta 1990;190:47-56.

13. Soleimani R, Favresse J, Roy T, Gruson D, Fillee C. Macro vitamin B12: an underestimated threat. Clin Chem Lab Med 2020;58: 408-15.

14. Belaidi N, Georges A, Lacroix I, Croisonnier A, Ducros V, Souberbielle JC, et al. Hypercalcemia and elevated concentration of vitamin D: a case report too easy to be true. Clin Chim Acta 2016;457:123-4. 
15. Ong MW, Salota R, Reeman T, Marta L, Jones L. Artifactual $25-\mathrm{OH}$ vitamin D level in multiple myeloma. Ann Clin Biochem 2017 Nov; 54:716-20.

16. Jones G. Pharmacokinetics of vitamin D toxicity. Am J Clin Nutr 2008;88:582S-6S.

17. Cavalier E, Lukas P, Bekaert AC, Carlisi A, Le Goff C, Delanaye P, et al. Analytical and clinical validation of the new Abbot Architect
25(OH)D assay: fit for purpose? Clin Chem Lab Med 2017;55: 378-84.

18. Siegel RL, Miller KD, Jemal A. Cancer statistics. CA Cancer J Clin 2016;2016:7-30.

19. Kyle RA, Therneau TM, Rajkumar SV, Larson DR, Plevak MF, Offord $J R$, et al. Prevalence of monoclonal gammopathy of undetermined significance. N Engl J Med 2006;354:1362-9. 